\title{
Mathematical modelling and optimization of the electroplating process with a rotating cathode to reduce the non-uniformity of the coating thickness
}

\author{
Denis Solovjev ${ }^{1,2, *}$, Inna Solovjeva², and Viktoriya Konkina ${ }^{2}$ \\ ${ }^{1}$ Tambov State University named after G.R. Derzhavin, 392036, Internatsionalnaya, 33, Tambov, \\ Russia \\ ${ }^{2}$ Tambov State Technical University, 392000, Sovetskaya, 106, Tambov, Russia
}

\begin{abstract}
The article is devoted to the development of an approach to improving the electroplating uniformity on the cathode surface, rotating around its axis, using a figured anode. A mathematical model of a stationary process with distributed coordinates, based on the equations of theoretical electrochemistry, has been developed for this approach. The problem for optimizing the anode shape according to the criterion for minimizing the non-uniformity of the deposited coating on the rotating cathode is formulated. The results of solving this problem are demonstrated on the example of the Zinc plating. Possible improvements to improve the accuracy of the calculation results for the developed mathematical model are given in the conclusion of the article.
\end{abstract}

\section{Introduction}

Obtaining a uniform coating layer on a product of complex geometric configuration is one of the most difficult problems encountered when electroplating is applied [1]. Optimal technological conditions and geometrical factors during the course of the electroplating process can reduce the non-uniformity of the coating layer on the processed product [2]. The current density [3], temperature [4] and acidity of the electrolyte [5] are technological conditions. In addition, non-stationary electrolysis modes are used [6, 7]: reversible, pulsed and asymmetric current. These modes provide for changing the shape and parameters of the current in order to slow down the growth of the metal coating in areas with higher current densities, providing an opportunity to obtain better cathode coatings than during electrolysis with direct current. Giving the anode a shape that reproduces the outlines of the surface of the product, refers to the geometric factors [8]. Apart from the above, additional cathodes [9] and non-standard baths [10] are used. A significant part of the products, to which electroplating coatings are required, has the form of rotation bodies. In order to simplify the process of electrolysis, as well as to improve the quality of the deposited coating, instead of the fixed placement of parts inside the cylindrical anode in an

\footnotetext{
* Corresponding author: solovjevdenis@mail.ru
} 
electroplating bath, use the following approach. The product is continuously rotated around its axis during the electrolysis process, and the anode is static and has the shape of a rod or plate [11]. The coating thickness along the radius of any cross-section is almost the same with a significant number of rotations. If the radius of the product along the axis of symmetry is not the same, then to achieve uniformity of the coating along the axis, an anode in the form of a curved rod or plate is required. It is extremely difficult to find a suitable form of the anode without experimental researches [12]. It should be noted that the formulation of experiments requires high time and financial costs. The use of computer simulation is a way out from the current situation [13]. In turn, numerical solutions to optimize the shape of the figure anode can only be obtained using a mathematical model of an electroplating process with a rotating cathode.

The purpose of the article is the mathematical modelling and optimization of the electroplating process with a rotating cathode to reduce the non-uniformity of the coating thickness.

\section{Development of a mathematical model for an electroplating process with a rotating cathode}

We introduce some conventions and designations. The bath space with electrolyte and electrodes located in it is an area in the form of a rectangular parallelepiped. A Cartesian coordinate system has been introduced, the center of which is aligned with the lower corner of the inner rectangular area of the electroplating bath. The length, width and height of the electroplating bath inner area are labelled $L_{x}, L_{y}$ and $L_{z}$ respectively. The abscissa axis of the coordinate system is directed along the length, the ordinate axis is directed along the width, and the applicate axis is directed along the height of the electroplating bath inner area. The cathode has the shape of a rotation body, the axis of which is located along the applicate axis. The length of the anode coincides with the length of the inner area $L_{x}$. The height of the anode is limited to coordinates $z_{a \min }$ below and $z_{a \max }$ above. The length of the cathode $l_{c}$ may be less than $L_{x}$. The abscissa of the end face closest to the plane 0zy is labelled as $x_{c}$. The axis of cathode rotation has ordinate $y_{c}$ and applicate $z_{c}$. The cathode radius is variable and is given by the abscissa function $r(x)$. The entire space between the walls, the bottom and the electrolyte mirror inside the electroplating bath is divided into several areas dependent on each other. This area is labelled as $V$. The surface located on the outside of the area $V$ is labelled as $S$. These areas are described by the following equations:

$$
\begin{gathered}
V=\left\{(x, y, z): 0 \leq x \leq L_{x} \cap 0 \leq y \leq L_{y} \cap 0 \leq z \leq L_{z}\right\}, \\
S=\left\{(x, y, z): x=0 \bigcup x=L_{x} \cup y=0 \cup y=L_{y} \cup z=0 \bigcup z=L_{z}\right\} .
\end{gathered}
$$

The assumption of a rectangular inner area for the bath is essential because it can affect the type of the equations that describe the insulator, anode and cathode areas.

The set of all points that determine the position of the anode, labelled as $V_{a}$. The anode surface is labelled as $S_{a}$ :

$$
\begin{gathered}
V_{a}=\left\{(x, y, z): 0 \leq x \leq L_{x} \cap z_{a \min } \leq z \leq z_{a \max } \cap y_{a}(x) \leq y \leq L_{y}\right\}, \\
S_{a}=\left\{(x, y, z): 0 \leq x \leq L_{x} \cap\left(y=y_{a}(x) \cap z_{a \min } \leq z \leq z_{a \max } U .\right.\right. \\
\left.\left.\cup y_{a}(x) \leq y \leq L_{y} \cap\left(z=z_{a \min } \cup z=z_{a \max }\right)\right)\right\}
\end{gathered}
$$

where $y_{a}(x)$ is the function defining the anode boundary. 
The function $y_{a}(x)$ is specified by linear interpolation at given points $\left(x_{0}, y_{0}\right),\left(x_{1}, y_{1}\right)$, $\ldots,\left(x_{N}, y_{N}\right)$ :

$$
y_{a}(x)=\frac{x-x_{i-1}}{x_{i}-x_{i-1}} y_{i}+\frac{x_{i}-x}{x_{i}-x_{i-1}} y_{i-1}, \quad x_{i-1} \leq x \leq x_{i}, \quad i=\overline{1, N}
$$

where $x_{0}=x_{c}$ and $x_{N}=x_{c}+l_{c}$.

The set of all points that determine the position of the cathode, labelled as $V_{c}$. The cathode surface is labelled as $S_{c}$ :

$$
\begin{gathered}
V_{c}=\left\{(x, y, z): x_{c} \leq x \leq x_{c}+l_{c} \cap \Delta y^{2}+\Delta z^{2} \leq r^{2}(x)\right\}, \\
S_{c}=\left\{(x, y, z): x_{c} \leq x \leq x_{c}+l_{c} \cap \Delta y^{2}+\Delta z^{2}=r^{2}(x) \cup\right. \\
\left.\cup\left(x=x_{c} \cup x=x_{c}+l_{c}\right) \cap \Delta y^{2}+\Delta z^{2} \leq r^{2}(x)\right\},
\end{gathered} .
$$

where $\Delta y=y-y_{c}$ is the difference between the current ordinate and the ordinate of the product axis; $\Delta z=z-z_{c}$ is the difference between the current applicate and the applicate of the product axis.

Since the cathode is a body of rotation and rotates around its axis of symmetry, areas $V_{c}$ and $S_{c}$ don't change over time.

The electrolyte area $V_{e l}$ and the insulator surface $S_{i n s}$ are labelled by the following sets:

$$
\begin{aligned}
& S_{\text {ins }}=\left\{(x, y, z):(x, y, z) \in S \cap(x, y, z) \notin\left(V_{a} \cup V_{c}\right)\right\}, \\
& V_{e l}=\left\{(x, y, z):(x, y, z) \in V \cap(x, y, z) \notin\left(S_{\text {ins }} \cup V_{a} \cup V_{c}\right)\right\} .
\end{aligned}
$$

According to assumption 10, the thickness of the coating doesn't depend on the rotational speed, but depends on the section determined by the abscissa value, and can be calculated based on the distribution of the instantaneous deposition rate at arbitrary point in time by the formula:

$$
\delta(x)=\frac{T}{2 \pi} \int_{0}^{2 \pi} v\left(x, y_{K}+r(x) \cos \alpha, z_{K}+r(x) \sin \alpha\right) d \alpha,
$$

where $x \in\left[x_{c}, x_{c}+l_{c}\right] ; T$ is the duration of the electroplating process; $\alpha$ is the angle of inclination $r(x)$ to the rotation axis of the product.

The instantaneous deposition rate at a given point on the cathode surface is defined as the ratio of the coating thickness, determined according to Faraday's law, to the time interval:

$$
v(x, y, z)=\frac{k \eta}{\rho} i_{c}(x, y, z),(x, y, z) \in S_{c},
$$

where $k$ is the electrochemical equivalent of a metal; $\rho$ is the density of the coating metal; $\eta$ is the cathode current output; $i_{c}(x, y, z)$ is the current density on the cathode surface.

The instantaneous deposition rate distribution of the coating over the boundary of the cross section perpendicular to the cathode axis is shown in fig. 1. 


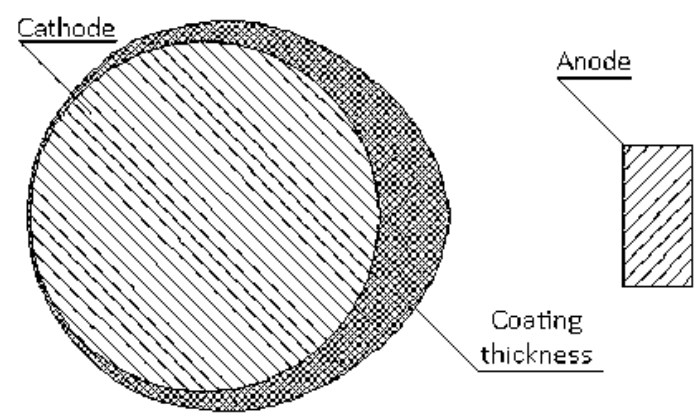

Fig. 1. Illustration to the instantaneous deposition rate distribution of the coating along the boundary of the cathode cross section

The current density can be found from Ohm's law in differential form. Anode current density $i_{a}$ is defined as the current density through the surface of the anode, cathode current density $i_{c}$ is defined as the current density through the surface of the cathode:

$$
\begin{aligned}
& i_{a}(x, y, z)=-\chi\left(\nabla \varphi(x, y, z), \vec{n}_{a}(x, y, z)\right),(x, y, z) \in S_{a}, \\
& i_{c}(x, y, z)=-\chi\left(\nabla \varphi(x, y, z), \vec{n}_{c}(x, y, z)\right),(x, y, z) \in S_{c},
\end{aligned}
$$

where $\chi$ is the electrical conductivity of the electrolyte; $\vec{n}_{a}, \vec{n}_{c}$ are the normals to the anode and cathode surface; $\nabla \varphi(x, y, z)$ is the gradient of the electric field potential at the point with $(x, y, z)$ coordinates.

The distribution of the electric potential $\varphi$ in the electrolyte area $V_{e l}$ can be found from the Laplace equation [14]:

$$
\frac{\partial^{2} \varphi(x, y, z)}{\partial x^{2}}+\frac{\partial^{2} \varphi(x, y, z)}{\partial y^{2}}+\left.\frac{\partial^{2} \varphi(x, y, z)}{\partial z^{2}}\right|_{V_{e l}}=0 .
$$

At the boundaries of the electrolyte area, the electric field is specified by the boundary conditions for equation (14) listed below.

Since the current doesn't flow through the insulator, the derivative along the normal $\vec{n}_{\text {ins }}$ according to Ohm's law is zero:

$$
\left.\frac{\partial \varphi(x, y, z)}{\partial \vec{n}_{i n s}}\right|_{S_{\text {iss }}}=0 .
$$

At the electrolyte points bordering the anode, the electric potential is less than the anode potential by an amount of $F_{a}$ depending on the anode current density:

$$
\varphi(x, y, z)+\left.F_{a}\left(i_{a}(x, y, z)\right)\right|_{s_{a}}=U,
$$

where $U$ is the voltage between anode and cathode.

In close proximity to the cathode surface in the electrolyte, the potential is greater than the cathode potential by an amount of $F_{c}$, depending on the cathode current density:

$$
\varphi(x, y, z)-\left.F_{c}\left(i_{c}(x, y, z)\right)\right|_{S_{c}}=0 .
$$




\section{Formulation of the optimization problem and choosing an algorithm for solving it}

The optimization problem is formulated as follows. Search the values of coordinates $y_{i}, i=$ $0, \ldots, N$, for which the criterion of non-uniformity $R$ is minimal:

$$
R=\frac{1}{l_{c}} \int_{0}^{l_{c}} \frac{\delta(x)-\delta_{\min }}{\delta_{\min }} \sqrt{1+\left(\frac{d r(x)}{d x}\right)^{2}} d x \rightarrow \min ,
$$

where $\delta_{\min }$ is the minimum value of coating thickness.

The search for coordinates $y_{i}, i=0, \ldots, N$ are carried out using (1) - (17) and the following constraints:

$$
\begin{array}{r}
y_{c}+r(x) \leq y_{a}(x) \leq L_{y}-d_{\text {min }}, x \in\left[x_{c}, x_{c}+l_{c}\right], \\
y_{c} \leq y_{a}(x) \leq L_{y}-d_{\text {min }}, x \in\left[0, x_{c}+l_{c}\right] \cup\left[x_{c}+l_{c}, L_{x}\right],
\end{array}
$$

where $d_{\min }$ is the minimum value of anode thickness.

The mathematical model calculation represented by (1) - (17) can occur for a considerable time depending on the dimension of the problem, which is determined by the grid step and distance between the electrodes [15]. This circumstance greatly complicates the use of gradient optimization methods, since a double recalculation of the coating thickness distribution is necessary to calculate the derivative [16]. In addition, the error in calculating the derivative increases significantly due to the approximation of the anode nodal points coordinates to the grid nodes, i.e. the minimum increment of the argument is the grid step. Using values close to the increments of the argument in calculating derivatives (i.e. order values of $10^{-6}$ ) as a grid step is obviously unacceptable. An attempt to reduce the search time for an extremum due to a relatively small number of iterations using second-order optimization methods based on calculating a matrix of second derivatives doesn't give the desired results, due to an even greater error in their calculation. The method of local variations is proposed to be used for solving problem (18). The method will avoid the need to calculate derivatives in order to determine the direction of the fastest movement to a minimum and at the same time preserve the possibility of a fairly rapid movement towards it.

\section{Materials and methods}

The solution of the optimizing problem anode shape will be considered on the example of the Zinc plating. The values list of constants and mode parameters that were used in the process of calculating the mathematical model is presented in Table 1.

Table 1. The values list of constants and mode parameters.

\begin{tabular}{|c|c|c|c|c|c|c|}
\hline $\begin{array}{c}\text { Symbol, } \\
\text { unit of } \\
\text { measurement }\end{array}$ & $\rho, \mathrm{kg} / \mathrm{m}^{3}$ & $k, \mathrm{~kg} /(\mathrm{A} \cdot \mathrm{h})$ & $\eta$ & $\chi, 1 /(\Omega \mathrm{m})$ & $U, \mathrm{~V}$ & $\delta_{\min }, \mu \mathrm{m}$ \\
\hline Value & 7130 & $1.22 \cdot 10^{-3}$ & 0.98 & 8.3 & 5 & 20 \\
\hline
\end{tabular}

The values of the electrolyzer geometrical characteristics and the electrodes located in it are presented in Table 2 . 
Table 2. The values of the electrolyzer geometrical characteristics and the electrodes.

\begin{tabular}{|c|c|c|c|c|c|c|c|c|c|}
\hline $\begin{array}{c}\text { Symbol, } \\
\text { unit of } \\
\text { measurement }\end{array}$ & $L_{x}, \mathrm{~m}$ & $L_{y}, \mathrm{~m}$ & $L_{z}, \mathrm{~m}$ & $z_{a \min }, \mathrm{m}$ & $z_{a \max }, \mathrm{m}$ & $x_{c}, \mathrm{~m}$ & $y_{c}, \mathrm{~m}$ & $z_{c}, \mathrm{~m}$ & $I_{c}, \mathrm{~m}$ \\
\hline Value & 1 & 0.5 & 0.5 & 0.2 & 0.3 & 0.05 & 0.2 & 0.25 & 0.9 \\
\hline
\end{tabular}

The cathode radius is given by the following function:

$$
r(x)=\left\{\begin{array}{l}
0.075, \quad 0.1 \leq x \leq 0.4 \\
0.175-0.25 x, \quad 0.4<x<0.6 \\
0.025, \quad 0.6 \leq x \leq 0.9
\end{array}\right.
$$

The anodic and cathodic polarization functions are:

$$
\begin{gathered}
F_{a}\left(i_{a}(x, y, z)\right)=0.935 i_{a}(x, y, z), \\
F_{c}\left(i_{c}(x, y, z)\right)=-0.188+0.3 \ln \left(0.43 i_{c}(x, y, z)\right) .
\end{gathered}
$$

Seven variable points defining the anode shape were selected, which coordinates are presented in Table 3.

Table 3. The coordinates of variable points.

\begin{tabular}{|c|c|c|c|c|c|c|c|}
\hline $\boldsymbol{i}$ & 0 & 1 & 2 & 3 & 4 & 5 & 6 \\
\hline $\boldsymbol{x}_{\boldsymbol{i}}$ & 0 & 0.2 & 0.4 & 0.5 & 0.6 & 0.8 & 1 \\
\hline $\boldsymbol{y}_{\boldsymbol{i}}$ & 0.95 & 0.95 & 0.95 & 0.95 & 0.95 & 0.95 & 0.95 \\
\hline
\end{tabular}

\section{Results and discussion}

Coordinate values that minimize the criterion for non-uniformity coating (18) are presented in Table 4.

Table 4. The optimal coordinates of variable points.

\begin{tabular}{|c|c|c|c|c|c|c|c|}
\hline $\boldsymbol{i}$ & 0 & 1 & 2 & 3 & 4 & 5 & 6 \\
\hline $\boldsymbol{x}_{\boldsymbol{i}}$ & 0 & 0.2 & 0.4 & 0.5 & 0.6 & 0.8 & 1 \\
\hline $\boldsymbol{y}_{\boldsymbol{i}}$ & 0.47 & 0.47 & 0.48 & 0.42 & 0.32 & 0.33 & 0.34 \\
\hline
\end{tabular}

The plane section, which is parallel to the bath bottom and passes through the cathode center, is presented in fig. 2. 


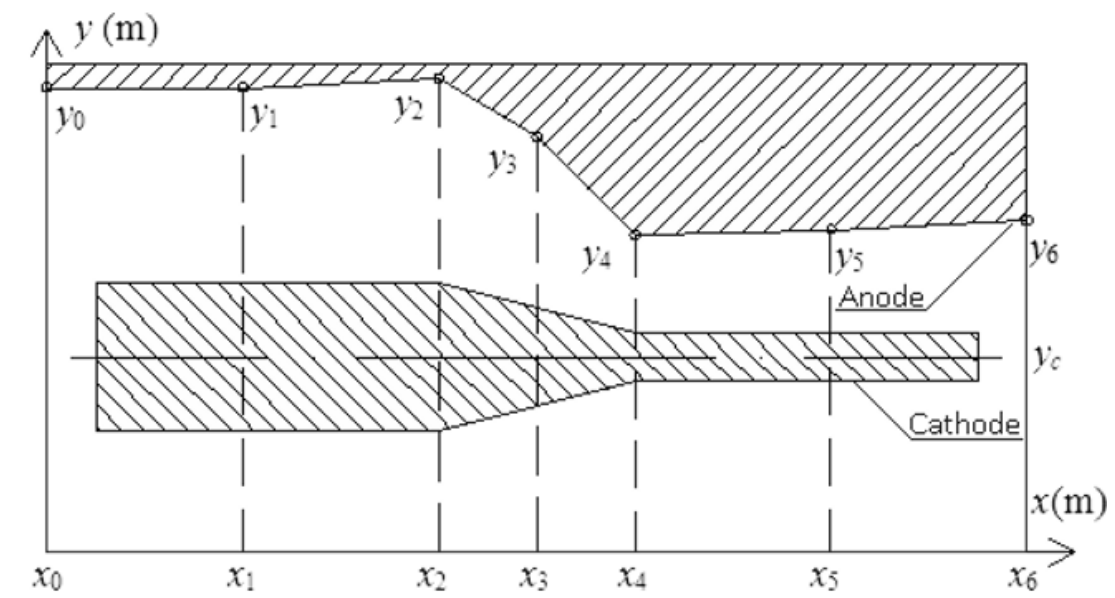

Fig. 2. Illustration to the plane section

The optimal anode shape for a given cathode shape in 3D is presented in fig. 3.

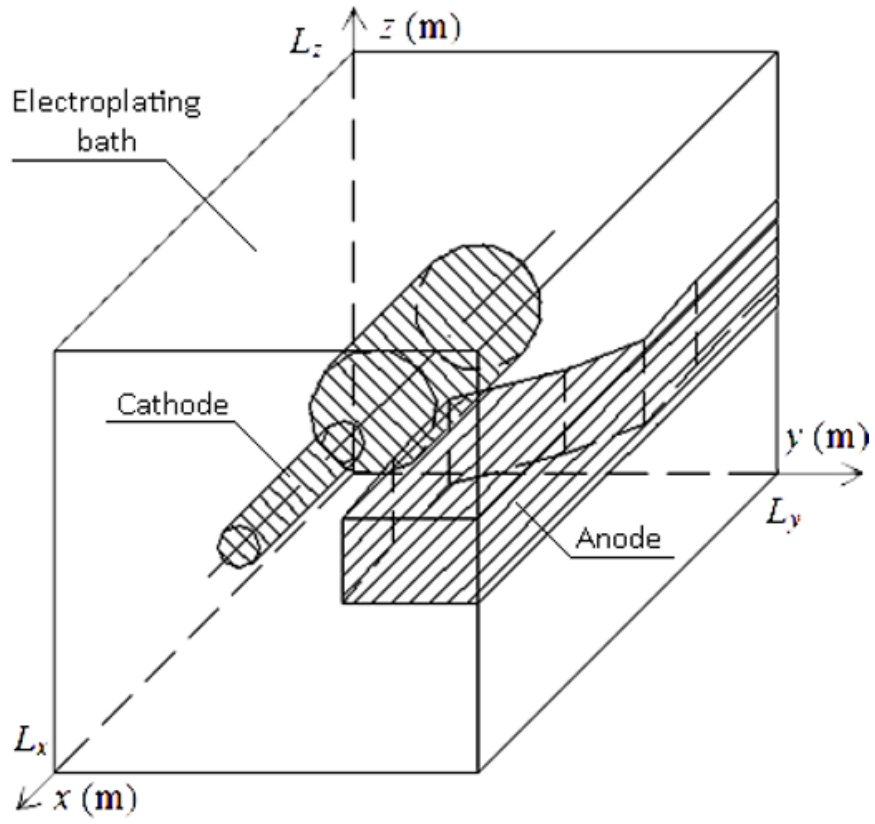

Fig. 3. Illustration to the optimal anode shape for a given cathode shape in 3D

The initial and optimal distribution of the coating thickness over the cathode length is presented in fig. 4. 


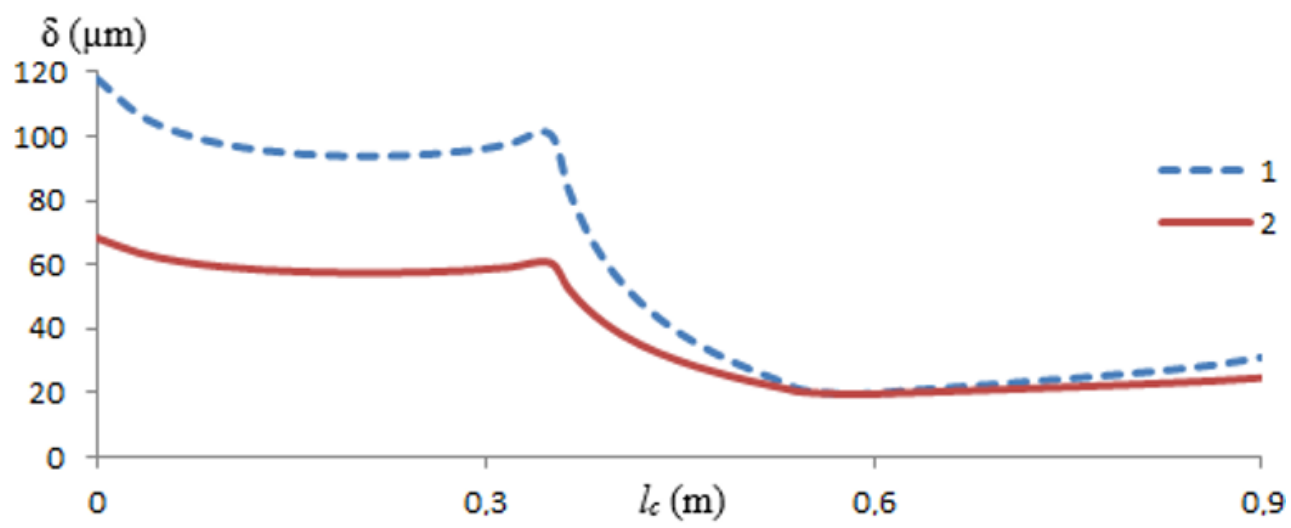

Fig. 4. Illustration to the initial (1) and optimal (2) distribution of the coating thickness

The criterion value for non-uniformity of coating (18) equal to 2.293 was obtained at the initial approximation of the variable coordinates of the anode nodal points. The nonuniformity coating criterion (18) decreased to a value of 1.412 as an optimization result. The non-uniformity coating criterion was reduced by $38.4 \%$ for the Zinc plating example considered.

\section{Conclusion}

The use of optimal shape anodes seems to be a promising direction for improving the uniformity of electroplating coatings on products. It is practically impossible to manually calculate the anode optimum shape, since the mathematical model of electroplating process contains a partial differential equation, an analytical solution of which can be obtained only for very simple cases. Therefore, an adequate mathematical description of such an electroplating process is crucial.

Possible improvements for the developed mathematical model in order to improve the accuracy of the results obtained with its help include:

- generalization of the problem formulation of searching the anode optimal shape with simultaneous search for the optimal control non-stationary electrolysis modes, e.g. reversible, pulsed and asymmetric current;

- other more suitable numerical methods can be used to interpolate the function of the cathode radius and the anode shape.

\section{References}

1. Y.D. Gamburg, G. Zangari, Theory and practice of metal electrodeposition (Springer, New York, 2011)

2. S. Kumar, S. Pande, P. Verma, Factor effecting electro-deposition process, Int. J. Curr. Eng. Technol., v. 5(2), pp. 700-703 (2015)

3. M.G. Pavlović, Lj.J. Pavlović, N.D. Nikolić, K.I. Popov, The effect of some parameters of electrolysis on apparent density of electrolytic copper powder in galvanostatic deposition, Materials Science Forum, v. 352, pp. 65-72 (2000)

4. S. Lambert, The influence of temperature on the efficiency of electroplating from various ionic liquids, Circuit World, v. 32(4), pp.36-41 (2006) 
5. B. Szczygiel, A. Laszczynska, Influence of bath concentration and $p H$ on electrodeposition process of ternary $\mathrm{Zn}-\mathrm{Ni}$-Mo alloy coatings, Transactions of the IMF, v. 92(4), pp. 196-202 (2014)

6. J.W. Dini, H.R. Johnson, The properties of gold deposits produced by DC, pulse and asymmetric AC plating, Gold Bull., v. 13(1), pp. 31-34 (1980)

7. A.V. Romanenko, Effect of reversing current parameters on the uniformity of zinc coatings obtained in ammonia electrolyte, Russ. J. Appl. Chem., v. 71(11), pp. 20202022 (1998)

8. Y.V. Litovka, A.M. Elizarov, A method for calculating the thickness of a coating on a complex-shaped electrode, Theor. Found. Chem. Eng., v. 37(1), pp. 88-91 (2003)

9. C.-W. Park, K.-Y. Park, An effect of dummy cathode on thickness uniformity in electroforming process, Results in Physics, v. 4(C), pp. 107-112 (2014)

10. H.L. de Maubeuge, Calculation of the optimal geometry of electrochemical cells: application to the plating on curved electrodes, J. Electrochem. Soc., v. 149(8), pp. 413-422 (2002)

11. Z.-W. Zhu, D. Zhu, N.-S. Qu, K. Wang, J.-M. Yang, Electroforming of revolving parts with near-polished surface and uniform thickness, Int. J. Adv. Manuf. Technol., v. 39(11-12), pp. 1164-1170 (2008)

12. M. Poroch-Seritan, S. Gutt, G. Gutt, I. Cojocaru, C. Cojocaru, T. Severin, Design of experiments for statistical modeling and multi-response optimization of nickel electroplating process, Chem. Eng. Res. Des., v. 89(2), pp. 136-147 (2011)

13. R.E. White, V.R. Subramanian, Mathematical modeling of electrodeposition, Plating \& Surface Finishing, v. 87(9), pp. 42-45 (2000)

14. H.P.E. Helle, G.H.M. Beek, J.Th. Ligtelijn, Numerical determination of potential distribution and current densities in multi-electrode systems, Corrosion, v. 37(9), pp. 522-530 (1981)

15. D.S. Solovjev, I.A. Solovjeva, Yu.V. Litovka, I.L. Korobova, About one counterexample of applying method of splitting in modeling of plating processes, J. Phys. Conf., v. 1015, 032138 (2018)

16. R. Fletcher, Practical Methods of Optimization (John Wiley \& Sons, Chichester, 1987) 